\title{
Letter
}

\section{H-mode Power Threshold Studies in Mixed Ion Species Plasmas at ASDEX Upgrade}

\author{
U. Plank ${ }^{1,2}$, T. Pütterich ${ }^{1,2}$, C. Angioni ${ }^{1}$, M. Cavedon ${ }^{1}$, G. D. \\ Conway $^{1}$, R. Fischer ${ }^{1}$, T. Happel ${ }^{1}$, A. Kappatou ${ }^{1}$, R. M. \\ McDermott $^{1}$, P. A. Schneider ${ }^{1}$, G. Tardini ${ }^{1}$, M. Weiland ${ }^{1}$ and \\ the ASDEX Upgrade Team $\dagger$ \\ ${ }^{1}$ Max-Planck-Institut für Plasmaphysik, Boltzmannstraße 2, D-85748 Garching \\ ${ }^{2}$ Ludwig-Maximilians-Universität München, Fakultät für Physik, Schellingstraße 4, \\ D-80799 München \\ E-mail: Ulrike.Plank@ipp.mpg.de
}

28 May 2020

\begin{abstract}
.
Understanding the dependence of the H-mode power threshold, $P_{\mathrm{LH}}$, on the main ion composition is crucial for H-mode operation in ITER. In this work, H-mode power threshold studies conducted at ASDEX Upgrade are presented. These were performed in hydrogen plasmas with helium doping and in mixed hydrogen-deuterium plasmas. $P_{\mathrm{LH}}$ stays constant in hydrogen plasmas with helium doping up to $c_{\mathrm{He}}=20 \%$. Power balance calculations with the transport code ASTRA show that the edge ion heat flux at the L-H transition is independent of helium concentration and applied auxiliary heating, supporting that a critical edge ion heat flux is needed to enter H-mode. A non-linear dependence of $P_{\mathrm{LH}}$ on the relative hydrogen content is found. $P_{\mathrm{LH}}$ and the edge ion heat flux are constant at the level of pure deuterium for $n_{\mathrm{H}} /\left(n_{\mathrm{H}}+n_{\mathrm{D}}\right)$ between 0 and 0.5 and constant on the level of pure hydrogen for $n_{\mathrm{H}} /\left(n_{\mathrm{H}}+n_{\mathrm{D}}\right)$ between 0.8 and 1.
\end{abstract}

Submitted to: Nucl. Fusion

\section{Introduction}

For ITER operation in the high confinement mode (H-mode) it is important to understand the dependence of the H-mode power threshold, $P_{\mathrm{LH}}$, on the main ion composition. Not only will fusion operation be performed in mixed deuterium-tritium (D-T) plasmas, but also the pre-fusion power operation will be performed in helium (He) or, preferentially, in hydrogen $(\mathrm{H})$ plasmas. Access to $\mathrm{H}$-mode during this early stage

$†$ See the author list of H. Meyer et al. 2019 Nucl. Fusion 59112014 
would allow for important subsystem tests, such as edge-localized mode mitigation and suppression systems.

However, $P_{\mathrm{LH}}(H)$ is about two times higher than $P_{\mathrm{LH}}(D)$ [1-3]. Therefore, ways of decreasing $P_{\mathrm{LH}}(\mathrm{H})$ are desired to maximize the $\mathrm{H}$-mode operational window in ITER. This is particularly important during the first stage of pre-fusion power operation (PFPO-1), when only limited auxiliary heating, namely solely wave heating, will be available [4].

At JET a reduction of $P_{\mathrm{LH}}(\mathrm{H})$ by up to $40 \%$ is observed in neutral beam heated $\mathrm{H}$ plasmas when a He concentration of up to $10 \%$ is added. Moreover, in ion cyclotron resonance wave heated (ICRH) plasmas without He doping, a non-linear increase of $P_{\mathrm{LH}}$ with increasing relative hydrogen content, $n_{\mathrm{H}} /\left(n_{\mathrm{H}}+n_{\mathrm{D}}\right)$, is found [5]. Recently, dedicated experiments were performed at ASDEX Upgrade (AUG) to address these points. In the following we present the results obtained from the $P_{\mathrm{LH}}$ studies in $\mathrm{H}$ plasmas with He doping and in mixed hydrogen-deuterium (H-D) plasmas.

\section{Methodology}

\subsection{Experimental Approach}

The H-mode power threshold was measured in consecutive discharges in pure $\mathrm{H}$ plasmas $\left(n_{\mathrm{H}} /\left(n_{\mathrm{H}}+n_{\mathrm{D}}\right) \approx 0.95\right)$, into which an increasing amount of He was added. In a second sequence of discharges $n_{\mathrm{H}} /\left(n_{\mathrm{H}}+n_{\mathrm{D}}\right)$ was scanned by changing the hydrogenic species of the gas fuelling.

All experiments were performed at a plasma current of $800 \mathrm{kA}$ and a toroidal magnetic field of $2.5 \mathrm{~T}$ at the magnetic axis, with $\mathrm{q}_{95}=5$. Slow power ramps were designed to trigger the $\mathrm{L}-\mathrm{H}$ transition, i.e. the transition from the low confinement mode (L-mode) to H-mode. In figure 1 (a) and (b) the power ramps of two example discharges are shown.

Two different heating schemes were employed to influence the ratio of direct electron to ion heating: electron cyclotron resonance wave heating $(\mathrm{ECRH})$, which exclusively heats the electrons, and neutral beam injection (NBI), which, in these discharges, preferentially heated the ions. The ECRH power ramp was made of $200 \mathrm{~kW}$ steps central X2 heating (see figure 1 (a)) [6]. NBI blips of $2 \mathrm{MW}$ and a duration of $12 \mathrm{~ms}$ were programmed at the very end of each heating step for charge exchange recombination spectroscopy (CXRS) measurements [7].

The NBI power ramp as depicted in figure 1 (b) (smoothed over $50 \mathrm{~ms}$ ) was achieved by modulation of the neutral beams. The injected neutral species of the NBI was hydrogen for dominant $\mathrm{H}$ plasmas, i.e. $n_{\mathrm{H}} /\left(n_{\mathrm{H}}+n_{\mathrm{D}}\right)>0.5$, and deuterium for dominant $\mathrm{D}$ plasmas. In both power ramps the power steps were sufficiently long (150 ms in dominant $\mathrm{H}$ plasmas and $200 \mathrm{~ms}$ in dominant $\mathrm{D}$ plasmas) to reach steadystate conditions before the next heating step $\left(\tau_{\text {th }}(D) \approx 140 \mathrm{~ms}\right.$ and $\left.\tau_{\text {th }}(H) \approx 80 \mathrm{~ms}\right)$. In the NBI heated discharges $600 \mathrm{~kW}$ of central ECRH was also applied to avoid tungsten 

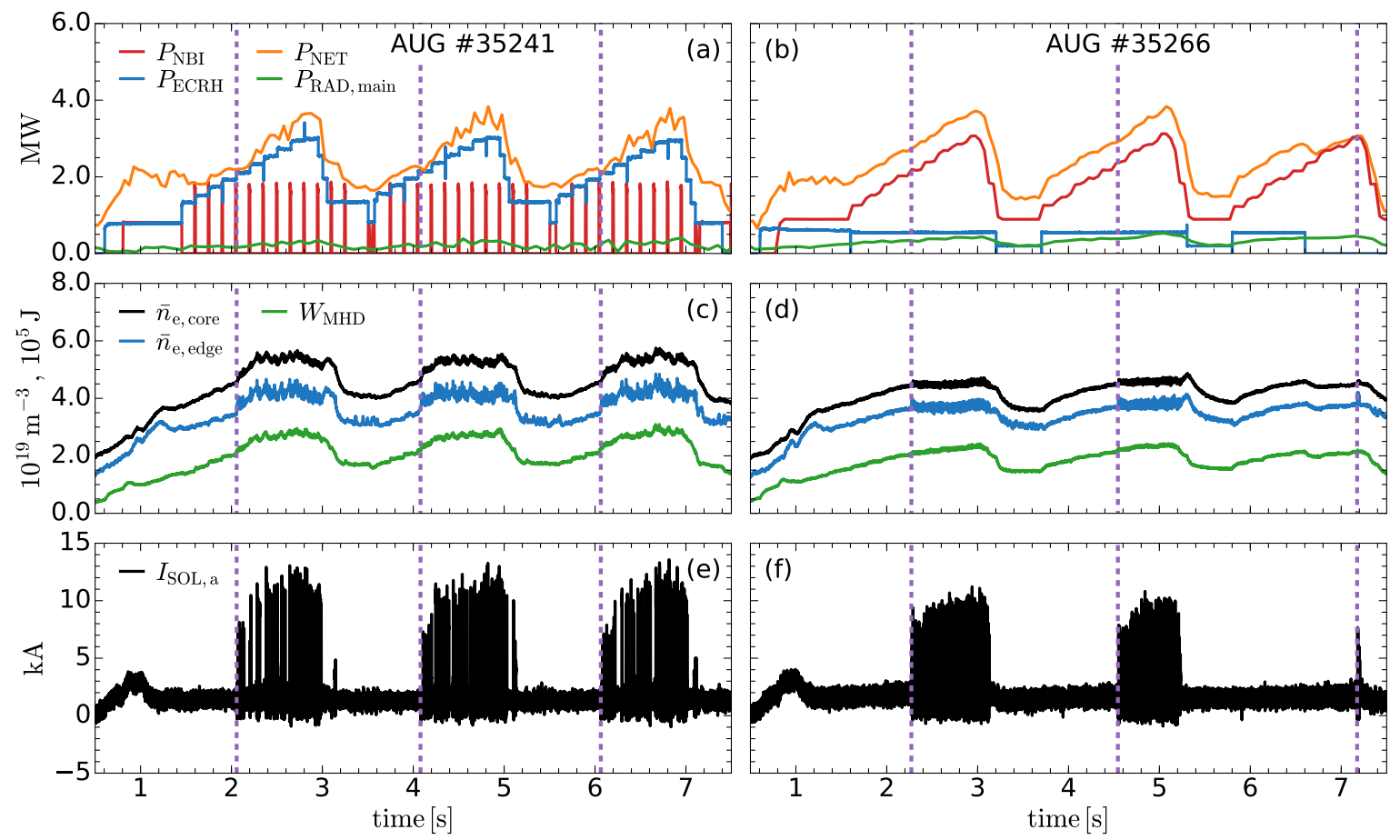

Figure 1. Time evolution of two example discharges used for $P_{\mathrm{LH}}$ studies in mixed ion species plasmas employing two different heating schemes: (a) ECRH and (b) NBI power ramp with total injected power and main chamber radiation reconstructed from bolometry, (c) and (d) line-integrated core and edge densities and stored energy, (e) and (f) shunt current signal of the outer divertor used for determination of the L-H transition (indicated by dashed lines).

(W) accumulation.

The $\mathrm{W}$ concentration at the $\mathrm{L}-\mathrm{H}$ transition was below the detection limit of $0.2 \times 10^{-5}$ in all discharges. Consequently, the plasma core radiation, $P_{\text {rad,main }}$ (see figure 1 (a) and (b)), reconstructed from bolometry [8], was low. At the L-H transition $P_{\text {rad,main }}$ varied between 200 and $500 \mathrm{~kW}$, which accounts for 10 to $20 \%$ of $P_{\mathrm{LH}}$. No systematic dependence of $P_{\text {rad,main }}$ on the applied external heating nor on the plasma composition was found, therefore, radiation losses were not taken into account in the determination of $P_{\mathrm{LH}}$.

$P_{\mathrm{LH}}$ is taken as the net input power, $P_{\text {net }}=P_{\text {heat }}-d W_{\mathrm{MHD}} / d t$ (see figure 1 (a) and (b)), averaged over $50 \mathrm{~ms}$ before the L-H transition, with $P_{\text {heat }}$ being the absorbed heating power from all the heating contributions with their respective losses and $W_{\text {MHD }}$ the plasma stored energy (see figure 1 (c) and (d)).

In all cases the L-H transition was triggered by a beam blip. Note, the beam blips delivered $2 \mathrm{MW}$ for about $12 \mathrm{~ms}$, but as the slowing down and the energy confinement time are much longer, the perturbation of $P_{\text {net }}$ due to the beam blip was on the order of 100 to $200 \mathrm{~kW}$, which is taken into account in the uncertainties of $P_{\mathrm{LH}}$.

The $\mathrm{L}-\mathrm{H}$ transition (indicated by vertical dashed lines in figure 1 ) is identified as the time point where the plasma starts to develop improved particle and energy confinement. 
This can be seen in the time traces as a sudden rise of the stored energy and the edge electron density (see figure 1 (c) and $(\mathrm{d})$ ). At this time point the plasma enters the oscillatory regime of L-H dithers, which is terminated by the first type-I ELM $[9,10]$. In all investigated discharges this dithering phase is short, so that the first type-I ELM occurs within the same power step or, the latest, in the following one. This implies that the power required to get the first type-I ELM is at most $200 \mathrm{~kW}$ higher than the here determined $P_{\mathrm{LH}}$. To pinpoint the $\mathrm{L}-\mathrm{H}$ transition, also time traces of magnetic measurements (Mirnov coils) and the shunt current signal of the outer divertor (see figure 1 (e) and (f)) were analysed, which both show the signature of the L-H dithers [11].

All discharges were performed close to or slightly above the density minimum of $P_{\text {LH }}$ to ensure efficient energy transfer between electrons and ions [12]. In AUG D and H plasmas the density minimum is at about $4.5 \times 10^{19} \mathrm{~m}^{-3}$ [13]. Feed forward gas puffing with puffing rates of $3 \times 10^{21} \mathrm{el} / \mathrm{s}(\mathrm{D})$ and $5 \times 10^{21} \mathrm{el} / \mathrm{s}(\mathrm{H})$ was used to get the desired densities (see figure 1 (c) and (d)).

For diagnostic purposes small amounts of nitrogen $(\mathrm{N})$ were injected into the

plasma, but not during the very first power ramp, such that a $\mathrm{N}$-free reference is available. The low-Z impurity content was monitored with CXRS [14], which delivered nitrogen and boron concentrations below $0.1 \%$. A $Z_{\text {eff }}$ measurement from visible Bremsstrahlung [15] gives radially averaged $Z_{\text {eff }}$ values of 1.2 to 1.3 for all discharges except for the discharges with He concentrations above $10 \%$ to $20 \%$. For the latter, $Z_{\text {eff }}$ increases from 1.5 up to 1.7 .

The He density was calculated from plume-corrected CXRS measurements [16]. The volume average of the He concentration, $c_{\mathrm{He}}=n_{\mathrm{He}} / n_{e}$, up to $\rho_{\text {tor }}=0.9$ was taken, as no strong gradients in $c_{\mathrm{He}}$ were observed. The relative hydrogen content within the confined plasma region was measured by means of a neutral particle analyser [?].

\subsection{Power Balance Calculations}

Following the findings from [12] and [17] that a critical value of the ion heat flux at the

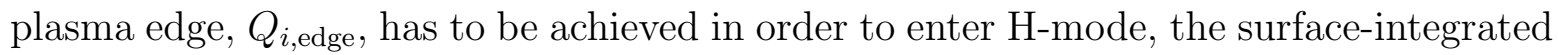
ion and electron heat fluxes, $Q_{i}$ and $Q_{e}$, evaluated at $\rho_{\text {tor }}=0.95$, were determined at the L-H transition by power balance analysis with the transport code ASTRA [18]. To compute the power deposition and, thus, the contribution of direct electron and ion heating by ECRH and NBI to $Q_{e}$ and $Q_{i}$, respectively, the microwave beam tracing code TORBEAM [19] and the real-time NBI code RABBIT [20] were coupled to ASTRA.

In the above described discharges an important contribution to $Q_{i}$ is the collisional heat exchange between electrons and ions, $p_{e i}$, where

$$
p_{e i} \propto n_{e}^{2} \frac{Z_{i}}{A_{i}} \frac{T_{e}-T_{i}}{T_{e}^{3 / 2}}
$$

with $n_{e}$ the electron density radial profile, $T_{e}$ the electron temperature radial profile and $T_{i}$ the ion temperature radial profile and $Z_{i}$ and $A_{i}$ are the charge and mass of the ion species, respectively. As can be seen from equation $1, p_{e i}$ strongly depends on the input 
electron and ion kinetic profiles, thus special care in their determination was taken. The $T_{e}$ and $n_{e}$ profiles, evaluated by integrated data analysis of several diagnostics [21], were averaged over $50 \mathrm{~ms}$ long time slices of the steady-state phases preceding the L-H transitions. For the $T_{i}$ profiles the CXRS measurements acquired during the last beam blip before the L-H transition were averaged.

The two hydrogenic species were considered as one species with an effective mass between one and two, depending on the measured $n_{\mathrm{H}} /\left(n_{\mathrm{H}}+n_{\mathrm{D}}\right)$. Furthermore, up to three impurity species densities were taken into account in the power balance calculations.

\section{Results}

\subsection{Power Threshold and Edge Ion Heat Flux in H Plasmas with He Doping}

Figure 2 (a) displays measurements of $P_{\mathrm{LH}}$ in $\mathrm{H}$ plasmas $\left(n_{\mathrm{H}} /\left(n_{\mathrm{H}}+n_{\mathrm{D}}\right) \approx 0.95\right)$ with increasing $c_{\mathrm{He}}$ from below $1 \%$ to almost $20 \%$, showing no dependence of $P_{\mathrm{LH}}$ on $c_{\mathrm{He}}$. This holds for both the NBI and the ECRH discharges, although for the former only the range of $c_{\mathrm{He}} \approx 1-5 \%$ could be investigated, while for the latter a fine scan of $c_{\mathrm{He}}$ up to $10 \%$ was performed. The line-integrated density varied in these experiments from 4.4 to $5.8 \times 10^{19} \mathrm{~m}^{-3}$, where the highest densities were reached in the discharges with the largest amount of He.

A comparison of $P_{\mathrm{LH}}$ with the respective value of $P_{\mathrm{scal}, 08}=0.0488 \bar{n}_{e}^{0.717} B_{\mathrm{tor}}^{0.803} S^{0.941}$, with $\bar{n}_{e}$ the line-averaged electron density in $10^{20} \mathrm{~m}^{-3}, B_{\text {tor }}$ the toroidal magnetic field at the magnetic axis in $\mathrm{T}$ and $S$ the plasma surface in $\mathrm{m}^{2}$ [22], is shown in figure 2 (b). Note, the fact that at the density minimum $P_{\mathrm{LH}}(\mathrm{H})$ matches $P_{\text {scal }, 08}$, which is computed for D plasmas, has been also reported earlier in [23] and will be discussed in section 3.2. Although the absolute values between the observed $P_{\mathrm{LH}}$ and the scaling do not agree, the density dependencies of $\mathrm{H}$ and $\mathrm{D}$ plasmas in the high density branch are well reproduced by the scaling [23]. Thus, taking the ratio $P_{\mathrm{LH}} / P_{\text {scal,08 }}$ adequately accounts for variations in the plasma density also in the conditions of the present experiments. However, as can be seen in figure 2 (b), no significant decrease of $P_{\mathrm{LH}}(\mathrm{H}) / P_{\mathrm{scal}, 08}$ with increasing $c_{\mathrm{He}}$ is found. This observation, namely that $P_{\mathrm{LH}}(\mathrm{H})$ stays constant with $c_{\mathrm{He}}$ up to $20 \%$, is in line with former observations at AUG. Then a decrease of $P_{\mathrm{LH}}(\mathrm{H})$ to $P_{\mathrm{LH}}(\mathrm{He})$, which is the same as $P_{\mathrm{LH}}(\mathrm{D})$ in $\mathrm{AUG}$, was only seen for $c_{\mathrm{He}}>30 \%$ [24].

The absolute values of $P_{\mathrm{LH}}(\mathrm{H})$, which are between 2.2 and $3 \mathrm{MW}$, are about $2 \times P_{\mathrm{LH}}(\mathrm{D})$ (see also figure $4(\mathrm{a})$ ), which is consistent with the simple isotope dependence of $P_{\mathrm{LH}}$ [1-3]. Figures 2 (a) and 2 (b) also show consistently higher values of $P_{\mathrm{LH}}(\mathrm{H})$ in the NBI heated discharges. An explanation for this increase of $P_{\mathrm{LH}}$ by $500 \mathrm{~kW}$ is found in the power balance calculations for these discharges, which show that to achieve the critical $Q_{i \text {,edge }}$ at the L-H transition, more NBI heating than ECRH is needed.

In figure 2 (c) $Q_{i \text {,edge }}$ at the $\mathrm{L}-\mathrm{H}$ transition is plotted against $c_{\mathrm{He}}$. It stays constant at a value of $2.2 \mathrm{MW}$, independent of the He concentration and the applied external 

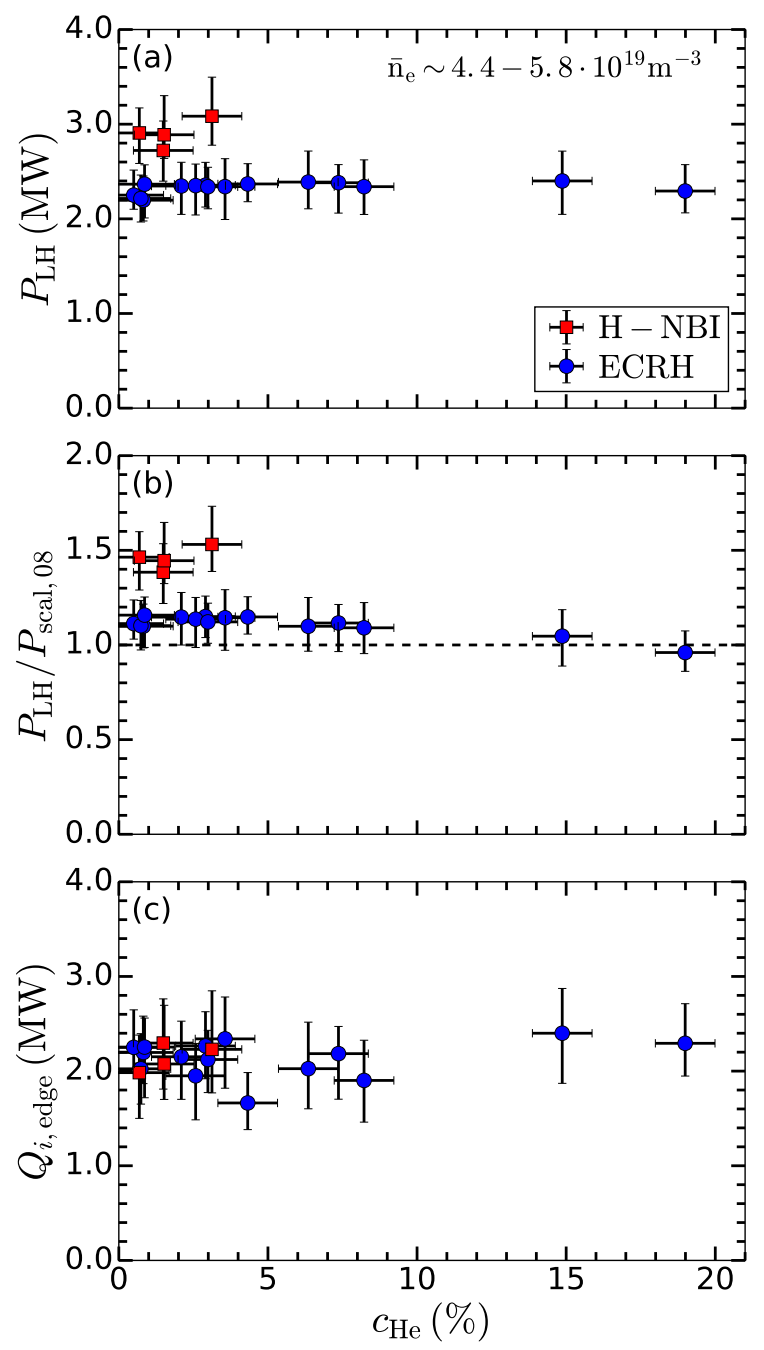

Figure 2. H-mode power threshold (a), divided by the H-mode power threshold in pure D plasmas derived from the multi-machine scaling presented in [22] (b) and edge ion heat flux at the L-H transition (c) in $\mathrm{H}$ plasmas with He doping versus the volumeaveraged He concentration.

heating. In contrast, the calculations show that $Q_{e, \text { edge }}$ is systematically lower for the ECRH than the NBI heated discharges at the L-H transition, what directly translates into the lower $P_{\mathrm{LH}}$. The counter intuitive observation that $Q_{e, \text { edge }}$ is smaller with ECRH than with NBI heating can be explained by $p_{\text {ei }}$ being the dominant contribution to $Q_{i}$, which is known to influence heat transport in hydrogen discharges [25].

From equation 1 it becomes clear that in $\mathrm{H}$ plasmas $p_{e i}$ is larger than in $\mathrm{D}$ plasmas due to the $Z_{i} / A_{i}$ dependence. Moreover, at the $\mathrm{L}-\mathrm{H}$ transition $\left(T_{e}-T_{i}\right) / T_{e}^{3 / 2}$ is in a regime where $p_{e i}$ increases with increasing $T_{e}$. Thus, at constant density, a reduction of $\left(T_{e}-T_{i}\right)$ leads to a reduction of $p_{e i}$. Figure 3 shows profiles of $Q_{i}$ (solid line) and $Q_{e}$ (dashed line) for an ECRH (a) and a NBI (b) heated discharge and their corresponding $Q_{e i}((\mathrm{c})$ and $(\mathrm{d}))$, the volume integral of $p_{\mathrm{ei}} \cdot Q_{e i}$ is calculated with the electron and ion temperature profiles depicted in figure 3 (e) and (f), respectively. With ECRH only 

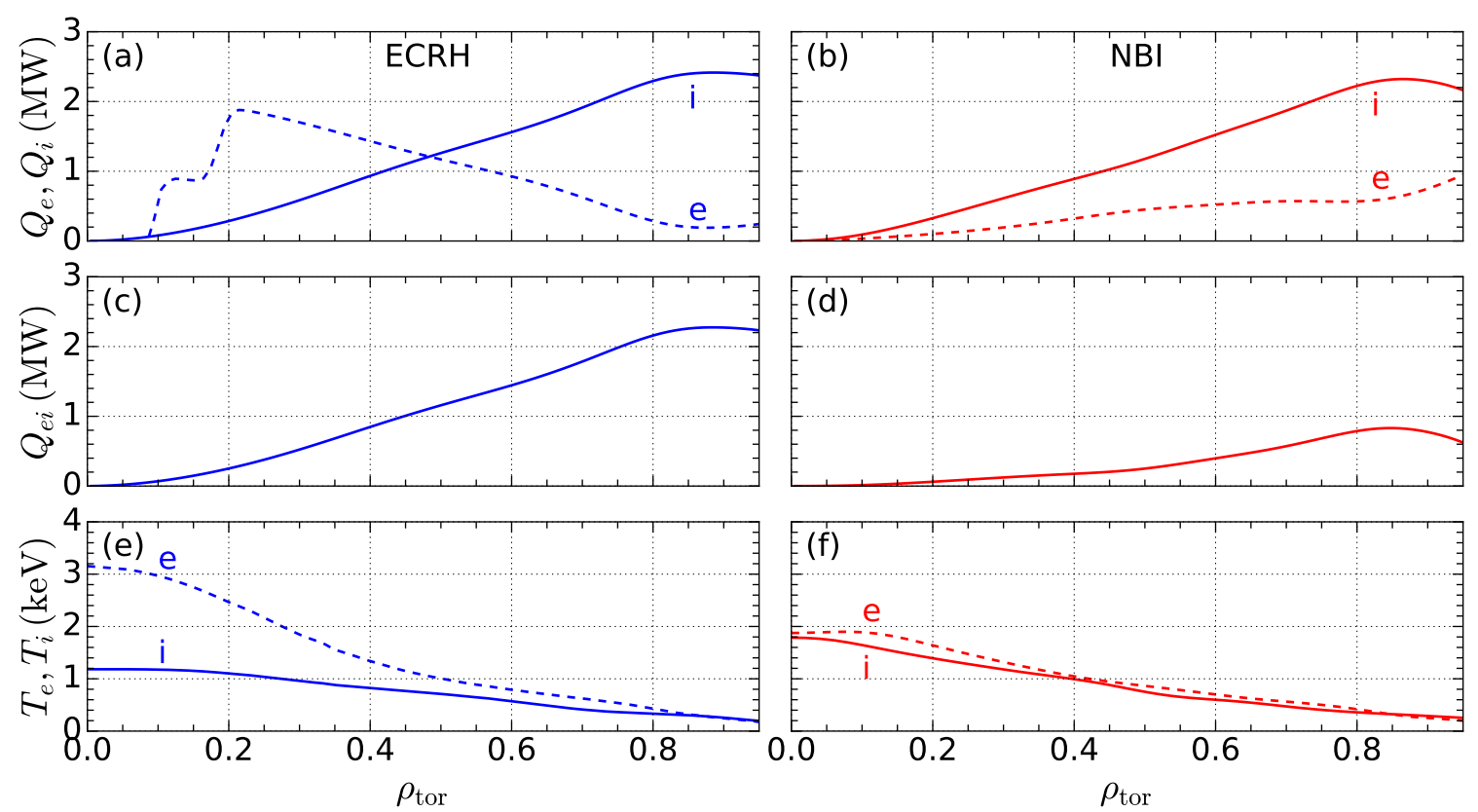

Figure 3. Radial profiles of the surface-integrated ion (solid) and electron (dashed) heat flux (a) and (b), the surface-integrated heat flux due to equipartition (c) and (d) and the input ion (solid) and electron (dashed) temperature profiles (e) and (f) for an ECRH and a NBI heated discharge, respectively.

the electrons are heated in the plasma center, hence $\left(T_{e}-T_{i}\right)$ is large and $Q_{e i}$ is the only contribution to $Q_{i}$, leading to a strong reduction of $Q_{e, \text { edge }}$. In contrast, in the NBI heated cases, also ions are heated directly and, additionally, the NBI exhibits a broad deposition profile, which leads to a decrease of $\left(T_{e}-T_{i}\right)$ over the whole radial range, thus, to a lower $Q_{e i}$ and, consequently, to a higher $Q_{e, \text { edge }}$.

Since at AUG it has been observed that $Q_{i \text {,edge }}$ is critical for the L-H transition [12], which is constant in these discharges, the lower $Q_{e, \text { edge }}$ in the ECRH compared to the NBI heated discharges leads to a lower $P_{\mathrm{LH}}$. Hence, the power balance calculations provide an explanation for a higher $P_{\mathrm{LH}}$ in $\mathrm{H}$ plasmas in NBI compared to ECRH discharges, which does not rely on differences in the toroidal rotation as found in [3].

The increase of $P_{\mathrm{LH}}$ due to increased plasma rotation caused by the co-current NBI torque input can not be excluded, since its influence on $Q_{i \text {,edge }}$ can not be disentangled from the effect of direct ion heating in the presented discharges. However, its contribution is within the error bars of the determination of $Q_{i \text {,edge }}$ : The total torque input introduced by the beam blips is negligible for the ECRH plasmas and the edge toroidal rotation, measured at $\rho_{\text {tor }}=0.95$, is between -5 and $5 \mathrm{~km} / \mathrm{s}$. In the case of the NBI heated plasmas the total torque input at the L-H transition is about $2 \mathrm{Nm}$ This leads to an edge toroidal rotation of 10 to $15 \mathrm{~km} / \mathrm{s}$, which, according to [12], would result in an increase of $P_{\mathrm{LH}}$ by 100 to $200 \mathrm{~kW}$.

These AUG $P_{\mathrm{LH}}$ studies can be directly related to the conditions during ITER PFPO-1, where H-mode operation in $\mathrm{H}$ plasmas at the density minimum is foreseen 
with dominant electron heating. The experiments suggest that admixture of $\mathrm{He}$ to $\mathrm{H}$ plasmas will not lower $P_{\mathrm{LH}}(H)$. On the other hand the findings underline again the importance of $Q_{i \text {,edge }}$ for the H-mode access. This gives more confidence to use the existing scaling of the density minimum, which is based on the assumption of a critical edge ion heat flux at the $\mathrm{L}-\mathrm{H}$ transition, for extrapolation the density minimum in ITER $[4,12]$. The observation that a critical $Q_{i \text {,edge }}$ at the $\mathrm{L}-\mathrm{H}$ transition is needed also implies that in plasmas where $Q_{e i}$ is the dominant contribution to $Q_{i}$, the transport properties of the confined plasma set the condition for the H-mode access. This is the case in the presented AUG experiments and will also be the case in ITER.
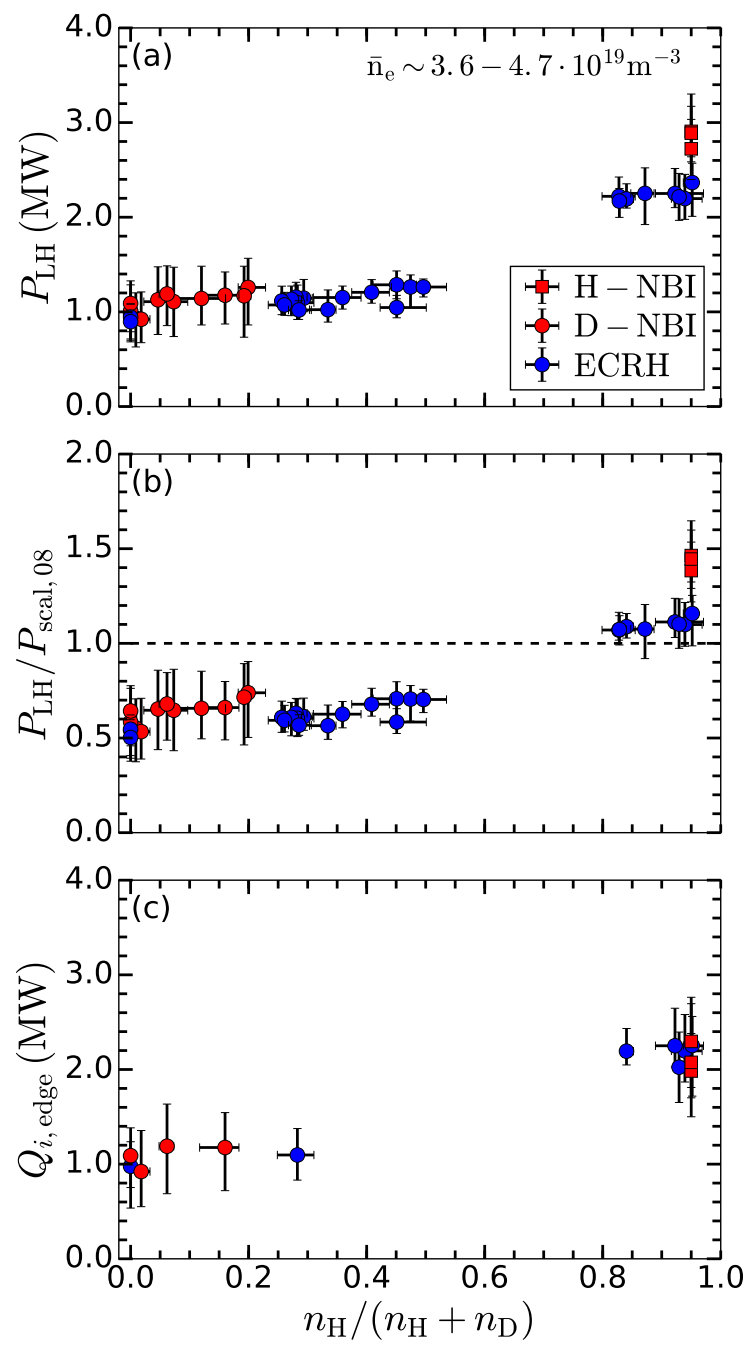

Figure 4. H-mode power threshold (a), divided by the H-mode power threshold in pure $\mathrm{D}$ plasmas derived from the multi-machine scaling presented in [22] (b) and edge ion heat flux at the L-H transition (c) in mixed hydrogen-deuterium plasmas versus the relative hydrogen content. 


\subsection{Power Threshold and Edge Ion Heat Flux in H-D Plasmas}

Figure 4 (a) shows the non-linear dependence of $P_{\mathrm{LH}}$ on $n_{\mathrm{H}} /\left(n_{\mathrm{H}}+n_{\mathrm{D}}\right)$. At AUG, $P_{\mathrm{LH}}$ remains constant at the level of pure $\mathrm{D}$ up to $n_{\mathrm{H}} /\left(n_{\mathrm{H}}+n_{\mathrm{D}}\right)=0.5$ and it is constant at the level of pure $\mathrm{H}$ for $n_{\mathrm{H}} /\left(n_{\mathrm{H}}+n_{\mathrm{D}}\right)>0.8$. These findings differ from the JET results, where a strong increase of $P_{\mathrm{LH}}$ was observed at $n_{\mathrm{H}} /\left(n_{\mathrm{H}}+n_{\mathrm{D}}\right)=0$ to 0.2 and $n_{\mathrm{H}} /\left(n_{\mathrm{H}}+n_{\mathrm{D}}\right)=0.9$ to $1[5]$.

The line-averaged density in the experiments varied from 3.6 to $4.7 \times 10^{19} \mathrm{~m}^{-3}$. To account for the density variation, the measured $P_{\mathrm{LH}}$ values were divided by $P_{\text {scal,08 }}$ from [22], which is shown in figure 4 (b). As can be seen in figure 4 (b), the non-linear dependence of $P_{\mathrm{LH}}$ on $n_{\mathrm{H}} /\left(n_{\mathrm{H}}+n_{\mathrm{D}}\right)$ does not change, but the absolute values show that the scaling of [22] overestimates $P_{\mathrm{LH}}$ in such a way that $P_{\mathrm{LH}}(\mathrm{H})$ is roughly at the level of $P_{\text {scal }, 08}(\mathrm{D})$ and $P_{\mathrm{LH}}(\mathrm{D})$ is overestimated almost by a factor of 2 .

This reduction of $P_{\mathrm{LH}}$ at AUG is favourable for ITER $P_{\mathrm{LH}}$ predictions, since it can be attributed to two effects, which also apply to ITER. First, the multi-machine scaling was determined for the high-density branch, i.e. for densities higher than the density minimum. The present experiments were conducted close to the density minimum of $P_{\mathrm{LH}}$ and there the scaling overestimates $P_{\mathrm{LH}}$ slightly (see also figure 5 in [23]). Second, the multi-machine scaling was mostly deduced from $P_{\mathrm{LH}}$ data in carbon wall tokamaks (about $11 \%$ of the selected data were from a metal wall device, namely Alcator C-Mod with a molybdenum wall) [22]. In 2007 AUG was equipped with a first wall and divertor surface completely made from tungsten and since then a reduction of $P_{\mathrm{LH}}(\mathrm{D})$ by up to $30 \%$ compared to the carbon wall was observed [23]. This reduction of $P_{\mathrm{LH}}$ is also seen at JET since the operation with the beryllium/tungsten (ITER-like) wall [26].

In D plasmas no difference of $P_{\mathrm{LH}}$ or $Q_{i \text {,edge }}$ between ECRH and NBI heated discharges is seen. This could be explained by the fact that $p_{e i}$ is less important in $\mathrm{D}$ plasmas. It is strongly reduced due to its $Z_{i} / A_{i}$ dependence, see equation 1. Consequently, there is no difference in $Q_{e, \text { edge }}$ at the L-H transition between ECRH and NBI heated discharges and, therefore, also not in $P_{\mathrm{LH}}$. On the other hand, in D plasmas also $P_{\mathrm{LH}}$ is so low that in NBI heated discharges no substantial external torque by the NBI is introduced (about $0.5 \mathrm{Nm}$ ). This makes that the differences in toroidal rotation at the L-H transition between ECRH and NBI heated discharges are small, and, thus, no difference in $P_{\mathrm{LH}}$ is observed between the two heating schemes. Toroidal velocity measurements at the plasma edge are, however, not available for these discharges.

Measurements of $P_{\mathrm{LH}}$ between $n_{\mathrm{H}} /\left(n_{\mathrm{H}}+n_{\mathrm{D}}\right)=0.5$ and 0.8 , the region where the transition from $P_{\mathrm{LH}}(\mathrm{D})$ to $P_{\mathrm{LH}}(\mathrm{H})$ can be expected to take place, have not been performed yet, but are planned for the upcoming hydrogen campaign at AUG.

\section{Summary and Outlook}

H-mode power threshold studies in mixed ion species plasmas, conducted at AUG, are presented. Experiments were performed in $\mathrm{H}$ plasmas with He concentrations of 1 to 
$20 \%$. A reduction of $P_{\mathrm{LH}}$ in $\mathrm{H}$ plasmas by He doping is not found, in contrast to recent observations at JET [5]. This holds for both ECRH and NBI heated plasmas, although for the latter $P_{\mathrm{LH}}(\mathrm{H})$ is higher by about $30 \%$.

Power balance calculations performed for all these discharges show that $Q_{i \text {,edge }}$ at the $\mathrm{L}-\mathrm{H}$ transition is independent of the He concentration and applied auxiliary heating, supporting the observation of Ryter et al. in [12] that a critical ion heat flux at the plasma edge is crucial for the L-H transition. From these calculations it is also seen that $Q_{e, \text { edge }}$ is higher in NBI compared to ECRH heated discharges, which can be ascribed to the dominant role of $p_{e i}$ to $Q_{i}$ under these experimental conditions. Thus, $P_{\mathrm{LH}}(\mathrm{H})$ is higher in NBI than in ECRH heated discharges, which is a complementary explanation to the effect of increased toroidal rotation [3].

In mixed H-D plasmas a non-linear dependence of $P_{\mathrm{LH}}$ on $n_{\mathrm{H}} /\left(n_{\mathrm{H}}+n_{\mathrm{D}}\right)$ is found. $P_{\mathrm{LH}}$ is constant at the level associated with pure $\mathrm{D}$ up to $n_{\mathrm{H}} /\left(n_{\mathrm{H}}+n_{\mathrm{D}}\right)=0.5$ and constant on the pure-H level for $n_{\mathrm{H}} /\left(n_{\mathrm{H}}+n_{\mathrm{D}}\right)>0.8$. Power balance calculations for a subset of the performed discharges show that $Q_{i \text {,edge }}$ also follows this behaviour. This dependence differs from the observed dependence at JET, where strongest changes of $P_{\mathrm{LH}}$ are mostly found at very low and very high $n_{\mathrm{H}} /\left(n_{\mathrm{H}}+n_{\mathrm{D}}\right)[5]$.

The AUG results suggest that $P_{\mathrm{LH}}$ is determined by the dominant plasma species, in contradiction to the observations made at JET, where the minority species strongly influences the behaviour of $P_{\mathrm{LH}}$. To give reliable predictions for ITER, further experiments are planned at AUG, which will extend the H-mode power threshold measurements and power balance calculations in mixed hydrogen-deuterium plasmas to the range $n_{\mathrm{H}} /\left(n_{\mathrm{H}}+n_{\mathrm{D}}\right)=0.5$ to 0.8 . Additional focus will be on the determination of edge electron and ion kinetic profiles, as well as radial electric field measurements in the plasma edge at the $\mathrm{L}-\mathrm{H}$ transition to improve the understanding of the $\mathrm{L}-\mathrm{H}$ transition and in particular its dependence on the isotope mass.

\section{Acknowledgment}

This work has been carried out within the framework of the EUROfusion Consortium and has received funding from the Euratom research and training program 2014-2018 and 2019-2020 under grant agreement No 633053. The views and opinions expressed herein do not necessarily reflect those of the European Commission.

\section{References}

[1] ASDEX Team. The H-mode of ASDEX. Nuclear Fusion, 29(11):1959-2040, Nov 1989.

[2] E Righi, D.V Bartlett, J.P Christiansen, G.D Conway, J.G Cordey, L.-G Eriksson, H.P.L. De Esch, G.M Fishpool, C.W Gowers, J.C.M. de Haas, P.J Harbour, N.C Hawkes, J Jacquinot, T.T.C Jones, W Kerner, Q.A King, C.G Lowry, R.D Monk, P Nielsen, F.G Rimini, G Saibene, R Sartori, B Schunke, A.C.C Sips, R.J Smith, M.F Stamp, D.F.H Start, K Thomsen, B.J.D Tubbing, and N Zornig. Isotope scaling of the H-mode power threshold on JET. Nuclear Fusion, 39(3):309-319, Mar 1999. 
H-mode Power Threshold Studies in Mixed Ion Species Plasmas at ASDEX Upgrade 11

[3] P. Gohil, T.C. Jernigan, T.H. Osborne, J.T. Scoville, and E.J. Strait. The torque dependence of the H-mode power threshold in hydrogen, deuterium and helium plasmas in DIII-D. Nuclear Fusion, 50(6):064011, May 2010.

[4] ITER Organization. ITER research plan within the staged approach (level III -provisional version). Technical Report ITR-18-003, ITER, 2018.

[5] J. Hillesheim, E. Delabie, E. R. Solano, C.F. Maggi, H. Meyer, E. Belonohy, I.S. Carvalho, E. de la Luna, A. Drenik, M. Gelfusa, C. Giroud, A.E. Hubbard, H.T. Kim, A. Huber, E. Lerche, B. Lomanowski, M. Mantsinen, S. Menmuir, I. Nunes, E. Peluso, F. Rimini, P.A. Schneider, M. Stamp, G. Verdoolaege, and JET Contributors. Implications of JET-ILW L-H transition studies for ITER. In 27th IAEA Fusion Energy Conference, 2018.

[6] J Stober, M Reisner, C Angioni, A Bañón Navarro, V Bobkov, A Bock, G Denisov, E Fable, R Fischer, G Gantenbein, L Gil, T Görler, V Igochine, W Kasparek, F Leuterer, A Litvak, R McDermott, A Meier, F Monaco, M Münich, V Nichiporenko, B Plaum, U Plank, E Poli, L Popov, Th Pütterich, Th Scherer, M Schubert, W Suttrop, E Tai, M Thumm, D Wagner, $\mathrm{H}$ Zohm, and and. Exploring fusion-reactor physics with high-power electron cyclotron resonance heating on ASDEX Upgrade. Plasma Physics and Controlled Fusion, 62(2):024012, Jan 2020.

[7] R. M. McDermott, A. Lebschy, B. Geiger, C. Bruhn, M. Cavedon, M. Dunne, R. Dux, R. Fischer, A. Kappatou, T. Pütterich, and E. Viezzer. Extensions to the charge exchange recombination spectroscopy diagnostic suite at ASDEX Upgrade. Review of Scientific Instruments, 88(7):073508, 2017.

[8] Pierre David, Matthias Bernert, Christoph Fuchs, Thomas Eich, and the ASDEX Upgrade Team. Optimisation of the radiated power computation at ASDEX Upgrade including local radiation. In preparation for Review of Scientific Instruments, 2020.

[9] G. D. Conway, C. Angioni, F. Ryter, P. Sauter, J. Vicente, and the ASDEX Upgrade Team. Mean and oscillating plasma flows and turbulence interactions across the L-H confinement transition. Phys. Rev. Lett., 106:065001, Feb 2011.

[10] G. Birkenmeier, M. Cavedon, G.D. Conway, P. Manz, U. Stroth, R. Fischer, G. Fuchert, T. Happel, F.M. Laggner, M. Maraschek, A. Medvedeva, V. Nikolaeva, D. Prisiazhniuk, T. Pütterich, F. Ryter, L.M. Shao, M. Willensdorfer, E. Wolfrum, and H. Zohm and. Magnetic structure and frequency scaling of limit-cycle oscillations close to L- to H-mode transitions. Nuclear Fusion, 56(8):086009, Jul 2016.

[11] M. Cavedon, G. Birkenmeier, T. Pütterich, F. Ryter, E. Viezzer, E. Wolfrum, R. Dux, T. Happel, P. Hennequin, U. Plank, U. Stroth, and M. Willensdorfer and. Connecting the global Hmode power threshold to the local radial electric field at ASDEX Upgrade. Nuclear Fusion, 60(6):066026, May 2020.

[12] F. Ryter, L. Barrera Orte, B. Kurzan, R.M. McDermott, G. Tardini, E. Viezzer, M. Bernert, R. Fischer, and the ASDEX Upgrade Team. Experimental evidence for the key role of the ion heat channel in the physics of the L-H transition. Nuclear Fusion, 54(8):083003, May 2014.

[13] F Ryter, M Cavedon, T Happel, R M McDermott, E Viezzer, G D Conway, R Fischer, B Kurzan, T Pütterich, G Tardini, and M Willensdorfer and. L-H transition physics in hydrogen and deuterium: key role of the edge radial electric field and ion heat flux. Plasma Physics and Controlled Fusion, 58(1):014007, Oct 2015.

[14] R M McDermott, R Dux, T Pütterich, B Geiger, A Kappatou, A Lebschy, C Bruhn, M Cavedon, A Frank, $\mathrm{N}$ den Harder, and E Viezzer and. Evaluation of impurity densities from charge exchange recombination spectroscopy measurements at ASDEX Upgrade. Plasma Physics and Controlled Fusion, 60(9):095007, Jul 2018.

[15] R. Fischer, S. K. Rathgeber, S. Fietz, J. Hobirk, A. Kallenbach, H. Meister, T. Pütterich, F. Ryter, G. Tardini, E. Wolfrum, H. Zohm, and ASDEX Upgrade Team. Effective ion charge Zeff from integrated analysis of multiple diagnostics at ASDEX Upgrade. In 37th EPS Conference on Plasma Physics, page P5.107, 2010.

[16] A Kappatou, R M McDermott, T Pütterich, R Dux, B Geiger, R J E Jaspers, A J H Donné, 
E Viezzer, M Cavedon, and the ASDEX Upgrade Team. A forward model for the helium plume effect and the interpretation of helium charge exchange measurements at ASDEX Upgrade. Plasma Physics and Controlled Fusion, 60(5):055006, Mar 2018.

[17] M. Schmidtmayr, J.W. Hughes, F. Ryter, E. Wolfrum, N. Cao, A.J. Creely, N. Howard, A.E. Hubbard, Y. Lin, M.L. Reinke, J.E. Rice, E.A. Tolman, S. Wukitch, Y. Ma, and and. Investigation of the critical edge ion heat flux for L-H transitions in Alcator C-Mod and its dependence on BT. Nuclear Fusion, 58(5):056003, Mar 2018.

[18] G.V.Pereverzev and P.N.Yushmanov. ASTRA - Automated System for TRansport Analysis. Technical Report IPP5/98, Max Planck Institute for Plasma Physics, 2002.

[19] E. Poli, A.G. Peeters, and G.V. Pereverzev. TORBEAM, a beam tracing code for electroncyclotron waves in tokamak plasmas. Computer Physics Communications, 136(1):90 - 104, 2001.

[20] M. Weiland, R. Bilato, R. Dux, B. Geiger, A. Lebschy, F. Felici, R. Fischer, D. Rittich, M. van Zeeland, the ASDEX Upgrade Team, and the Eurofusion MST1 Team. RABBIT: Real-time simulation of the NBI fast-ion distribution. Nuclear Fusion, 58(8):082032, Jul 2018.

[21] R. Fischer, C. J. Fuchs, B. Kurzan, W. Suttrop, E. Wolfrum, and ASDEX Upgrade Team. Integrated data analysis of profile diagnostics at ASDEX Upgrade. Fusion Science and Technology, 58(2):675-684, 2010.

[22] Y R Martin, T Takizuka, and the ITPA CDBM H-mode Threshold Data Group. Power requirement for accessing the H-mode in ITER. Journal of Physics: Conference Series, 123:012033, Jul 2008.

[23] F. Ryter, S.K. Rathgeber, L. Barrera Orte, M. Bernert, G.D. Conway, R. Fischer, T. Happel, B. Kurzan, R.M. McDermott, A. Scarabosio, W. Suttrop, E. Viezzer, M. Willensdorfer, and E. Wolfrum and. Survey of the H-mode power threshold and transition physics studies in ASDEX Upgrade. Nuclear Fusion, 53(11):113003, Sep 2013.

[24] F. Ryter, T. Pütterich, M. Reich, A. Scarabosio, E. Wolfrum, R. Fischer, M. Gemisic Adamov, N. Hicks, B. Kurzan, C. Maggi, R. Neu, V. Rohde, and G. Tardini and. H-mode threshold and confinement in helium and deuterium in ASDEX Upgrade. Nuclear Fusion, 49(6):062003, May 2009.

[25] P.A. Schneider, A. Bustos, P. Hennequin, F. Ryter, M. Bernert, M. Cavedon, M.G. Dunne, R. Fischer, T. Görler, T. Happel, V. Igochine, B. Kurzan, A. Lebschy, R.M. McDermott, P. Morel, M. Willensdorfer, and and. Explaining the isotope effect on heat transport in L-mode with the collisional electron-ion energy exchange. Nuclear Fusion, 57(6):066003, Apr 2017.

[26] C.F. Maggi, E. Delabie, T.M. Biewer, M. Groth, N.C. Hawkes, M. Lehnen, E. de la Luna, K. McCormick, C. Reux, F. Rimini, E.R. Solano, Y. Andrew, C. Bourdelle, V. Bobkov, M. Brix, G. Calabro, A. Czarnecka, J. Flanagan, E. Lerche, S. Marsen, I. Nunes, D. Van Eester, and M.F. Stamp and. L-H power threshold studies in JET with Be/W and C wall. Nuclear Fusion, 54(2):023007, Jan 2014. 